\title{
Call for Papers: Heft 4/2012
}

\section{Visuelle Soziologie}

Die Soziale Welt plant, Heft 4/2012 als ein Themenheft zu gestalten, das sich mit den Forschungen im Bereich der Visuellen Soziologie befasst. Visualität erfährt in der jüngeren Zeit eine sehr stark vermehrte Aufmerksamkeit quer durch die Sozial-, Geistes- und Kulturwissenschaften und reflektiert damit die gesteigerte gesellschaftliche Bedeutung der Kommunikation in und mit Bildern, Filmen und anderen visuellen Darbietungsformen. Seinen erkenntlich stärksten Niederschlag hat diese als ,visual turn' apostrophierte vermeintliche Kulturwende in den sogenannten ,Bildwissenschaften' gefunden, die damit zugleich das Projekt der transdisziplinären Etablierung einer neuen Wissenschaft vorantreibt.

Weniger als diese breiteren kulturwissenschaftlichen Entwicklungen steht hier indes die dezidiert soziologische Perspektive auf Bilder, Filme, Videos und andere Formen visueller Kommunikation im Vordergrund. Denn wenn im Zuge der ja kaum zu leugnenden wachsenden Durchdringung aller Lebenswelten mit Bildern ebenso neue Weisen des Umgangs damit erwachsen, die sich zu den etablierten Formen visuellen Verstehens, visueller Wissensproduktion und visueller Kommunikation gesellen, so eröffnen sich einer visuellen Soziologie neue Chancen. Dabei geht es nicht allein um die Herausforderungen und Möglichkeiten, die sich durch visuelle und audiovisuelle Daten für die Sozialforschung bieten. Es geht auch um die theoretische Reflexion der Implikationen des angenommenen kulturellen Wandlungsprozesses im Zuge des wachsenden Umfangs und Bedeutungszuwachses des Visuellen mit Blick auf die Frage, was das an unseren gegenwärtigen Arten und Weisen der Vergesellschaftung verändert.

Wir sprechen Kolleginnen und Kollegen an, die empirische Forschungsergebnisse und theoretische Positionen in diesem Kontext vorweisen können. Gedacht ist an Beiträge, die entweder prinzipiell methodische und methodologische Fragen im Umkreis der visuellen Soziologie stellen oder aber Forschungsergebnisse präsentieren und an ihnen die Spezifizität dieses Zugangs präsentieren, diskutieren, erläutern oder aber verwerfen.

Ein zweiseitiges Exposé des Aufsatzes sollte bis zum 31.8.2012 vorliegen. Die vollständigen Artikel können bis zum 15.11.2012 eingereicht werden. Die Beiträge sollten der Redaktion mit dem Vermerk „Themenheft Visuelle Soziologie“ gesendet werden. Es gelten die üblichen Regeln der Manuskriptgestaltung.

\section{Zum Verfahren:}

Die Soziale Welt ist eine Fachzeitschrift mit Peer-review-Verfahren. Von diesem Verfahren wird auch im Falle eines Themenheftes nicht abgewichen. Eingereichte Beiträge werden also einer Begutachtung zugeführt und nur im Falle eines positiven Begutachtungsverfahrens angenommen.

Bei Rückfragen stehen Ihnen die für dieses Themenheft federführenden Herausgeber Alejandro Baer (a.baer@soziologie.uni-muenchen.de) und Bernt Schnettler (schnettler@uni-bayreuth.de) zur Verfügung. 